\title{
Actividad física en residentes de medicina familiar de Bogotá y su recomendación a los pacientes
}

\section{Physical Activity in Bogotá Family Practice Residents and their recommendation to Patients}

\author{
Fecha de recepción: 08 Abril 2016 | Fecha de aprobación: 31 Mayo 2016
}

\author{
María Camila Barrios M. \\ Universidad de La Sabana, Colombia \\ FABio Andrés Gil M. \\ Juan Gabriel García M. \\ Alejandro Soler S. \\ JOHN DUPERly \\ Carlos Pinzón C.
}

Cómo citar: Barrios MC, Gil FA, García JG, Soler A, Duperly J, Pinzón C. Actividad física en residentes de medicina familiar de Bogotá y su recomendación a los pacientes. Univ Med. 2017; 58(3): 1-7. doi: https:// doi.org/10.11144/Javeriana.umed58-3.fami

\section{RESUMEN}

Objetivo: Identificar los hábitos de actividad física (AF) en residentes de medicina familiar de Bogotá y las recomendaciones que dan sobre esta a los pacientes. Metodología: Estudio de corte transversal con aplicación de parte de la encuesta de Healthy Doctor = Healthy Patient y preguntas sobre las "Características del médico y de la práctica clínica" de la iniciativa Exercise is Medicine ${ }^{\circledR}$ en los profesionales en formación en medicina familiar de 5 universidades de Bogotá. Resultados: El total de participantes fue de 97 residentes, quienes reportaron AF vigorosa, moderada y leve. Conclusiones: Los resultados evidenciados y comparados con la literatura internacional disponible (Reino Unido, Estados Unidos, Nueva Zelanda y Canadá) muestran que hay convergencia en la necesidad de capacitar médicos de atención primaria en la forma correcta de interrogar y recomendar la AF, de forma escrita, teniendo el formato incluido en la historia clínica esto respaldado por iniciativas internacionales.

Palabras clave

actividad física; ejercicio; medicina familiar.

\begin{abstract}
Objective: To identify habits and physical activity recommendations in family medicine residents of Bogota. Methodology: Cross-sectional study with application of the survey Healthy Doctor = Healthy Patient and using questions from Doctor Characteristics and of clinical practice from Exercise is Medicine ${ }^{\circledR}$ in professionals in training in family medicine in five universities of Bogotá. Results: The total number of participants was 97 residents. They reported vigorous, moderate, and mild physical activity. Conclusions: The results evidenced in this study and compared with international literature available (UK, USA, New Zealand, and Canada) show that there is convergence on the need to train primary care physicians with sufficient skills and knowledge for the proper and objective assessment and prescription of physical activity and structure a
\end{abstract}


service model in which physical activity is included within the medical history.

Keywords

Physical activity; exercise; family medicine.

\section{Introducción}

La medicina familiar es una especialización basada en el abordaje e intervención del paciente como un todo, de forma integral, pues se tienen en cuenta cada uno de los aspectos personales, familiares y comunitarios para lograr mantener la salud y prevenir la aparición de enfermedad. En relación con dicho alcance de la medicina familiar, es vital su relación con la prescripción de actividad física (AF), que exige recomendaciones e indicaciones claras, asequibles y con un importante sustento bibliográfico y evidencia mundial [1].

Muchos estudios clínicos han mostrado la AF como uno de los pilares en la prevención y manejo de las enfermedades crónicas no transmisibles, como las enfermedades cardiovasculares, la diabetes mellitus y el síndrome metabólico, que ocupan el primer puesto de causas de morbimortalidad en el mundo, con un $80 \%$ de muertes en países en vías de desarrollo [2]. Es claro que la $\mathrm{AF}$ debe ser recomendada por todos los médicos, sin importar su nivel o área de especialización a la que pertenezcan; sin embargo, son los médicos familiares quienes se enlistan de manera importante en el gran grupo de la atención primaria en salud, pues son los promotores de hábitos saludables como medio de prevención en el proceso salud-enfermedad [3].

En un estudio realizado en Estados Unidos con 4501 médicas, que buscaba indagar variables potencialmente relacionadas con conductas de prevención y tamizaje, se encontró que ser médico de atención primaria y la práctica de hábitos saludables fueron significativamente relacionados con la consejería reportada y el tamizaje realizado. Otras variables relacionadas fueron: región de procedencia, etnia, sitio de práctica y nivel de educación y entrenamiento del personal de salud [4].
Otro estudio realizado en médicos de atención primaria en 42 poblaciones rurales y urbanas de Nueva Zelanda evaluó la efectividad a largo plazo de la consejería y asesoría en AF. Se obtuvo como resultado un cambio significativo en la percepción de calidad de vida de los pacientes y en el nivel de AF posterior a la consejería [5]. Se suma a esta evidencia un estudio realizado en el Reino Unido, con 526 pacientes, en quienes se evaluó su interés en la realización de AF. Se encontró que casi al $100 \%$ de los pacientes reclutados les interesaba hacer algún tipo de AF; pero, a su vez, se evidenció la necesidad de mejorar la adherencia del personal de salud a los protocolos de medición objetiva de la AF y a su prescripción [6].

Sobre dicho contexto, se ha desarrollado la iniciativa Healthy Doctor=Healthy Patient, en cabeza de la doctora Erika Frank, y en Colombia de la mano del doctor John Duperly. La estrategia fundamenta su acción en el supuesto de demostrar que los hábitos que tenga el médico influyen directamente en las recomendaciones que este da a sus pacientes; es decir, aquel médico que se preocupa por realizar AF y lo logra es quien en sus consultas en mayor medida la prescribe y la recomienda [7]. El propósito principal de la iniciativa es trabajar e intervenir en los profesionales médicos, para lograr, por un lado, profesionales de la salud más saludables con una fuerte orientación y capacitación para la consejería sobre hábitos saludables; por otro, pacientes con mejor estilo de vida, menos enfermedades y mejor adherencia a las recomendaciones dadas por el profesional de la salud [8].

Teniendo en cuenta lo anterior, en Colombia no hay ningún estudio publicado sobre este tema enfocado en médicos residentes, por lo que resulta necesario evaluar si los médicos pertenecientes a los programas de formación en medicina familiar de Bogotá cumplen con un mínimo de $\mathrm{AF}$, conocer si el ambiente universitario los estimula a la realización de AF, establecer la forma y frecuencia de recomendación de $\mathrm{AF}$ a los pacientes, evaluar conocimientos mínimos generales sobre la prescripción de AF y conocer si se debe o no 
intervenir en los médicos familiares para mejorar su estilo de vida y, de esta manera, mejorar las recomendaciones dadas a los pacientes.

\section{Metodología}

Tipo de estudio

Estudio descriptivo de corte transversal.

\section{Población}

Médicos residentes de medicina familiar de los cinco programas que existen actualmente en Bogotá: Universidad de La Sabana, Pontificia Universidad Javeriana, Escuela de Medicina Juan N. Corpas, Fundación Universitaria de Ciencias de la Salud (FUCS) San José y Universidad El Bosque.

\section{Criterios de inclusión y exclusión}

Médicos residentes de medicina familiar que durante el desarrollo del estudio cursaran cualquiera de los tres años de formación en alguno de los cinco programas de posgrado en medicina familiar de Bogotá y que aceptaran voluntariamente participar en el estudio. Se excluyeron aquellos residentes con alguna discapacidad física que les impidiera realizar AF.

\section{Instrumento}

El instrumento de medición empleado consiste en un cuestionario autodiligenciable de forma física en el que se encuentran, por un lado, la sección de preguntas sobre AF de la encuesta Healthy Doctor=Healthy Patient, de la doctora Erika Frank [9], adaptado al español por el Grupo de Investigación en Enfermedades Crónicas y Estilo de Vida, a cargo del doctor John Duperly [8]; por otro, las preguntas sobre las "Características del médico y de la práctica clínica" de la iniciativa Exercise is Medicine ${ }^{\circledR}$ [6].
Con la aplicación del instrumento a los médicos residentes se evaluaron cinco aspectos: a) datos sociodemográficos, b) hábitos y comportamientos, c) ambiente universitario, d) conocimientos mínimos sobre $\mathrm{AF}$ según las recomendaciones dadas por el American College of Sport Medicine y e) práctica clínica [6]. Dentro del instrumento se aclaró a qué correspondían las diferentes intensidades de AF: vigorosa, para las que "requieren un esfuerzo físico fuerte, le hacen respirar y latir el corazón mucho más fuerte de lo normal"; moderada, "las que requieren un esfuerzo físico moderado, le hacen respirar algo más fuerte de lo normal", y leve, para "el tiempo que dedica caminando".

\section{Conducción del estudio}

Se realizó contacto telefónico y vía correo electrónico con los directores de los cinco programas de Especialización en Medicina Familiar de Bogotá, con los cuales se fijó día y hora donde estuvieran reunidos todos los residentes (día académico) de cada programa para la aplicación del instrumento. En cada encuentro con los diferentes grupos de residentes se explicaron verbalmente los objetivos y alcances del proyecto, se explicaron criterios de inclusión y exclusión y se obtuvo la firma de consentimiento informado previo a la aplicación del instrumento, con la asistencia de los investigadores durante su diligenciamiento. Los datos recolectados fueron transcritos a un formulario en Google Forms, obteniendo la base de datos en Excel para su análisis.

\section{Tamaño de la muestra}

Se estimó un tamaño de muestra basado en la prevalencia de la recomendación de $\mathrm{AF}$ en un 53 $\%$, de acuerdo con la literatura [10]. El tamaño de muestra fue estimado con un valor alfa de 0,05 y un parámetro beta de 0,80. El cálculo de tamaño muestral fue de 89 ; sin embargo, se evaluaron 97 de 130 (74,6\%) residentes de medicina familiar de la ciudad de Bogotá. 


\section{Análisis de la información}

Se realizó un análisis descriptivo de los datos. Para las variables continuas se estimaron las medias y desviaciones estándar para cada una de ellas, y para las variables categóricas se estimaron proporciones para cada una de las categorías de análisis. Todos los análisis se realizaron en el programa Stata 12.

\section{Aspectos éticos}

Con el fin de garantizar el rigor ético se tomó en cuenta la Declaración de Helsinki de la Asociación Médica Mundial, el Código de Núremberg y la Resolución 8430 de 1993 como pauta de las consideraciones éticas establecidas por la legislación colombiana. Según el artículo 10 de la resolución mencionada, se considera que nuestra investigación no es de riesgo, debido a que el cuestionario autodiligenciable no influye en cambios respecto a la patología del paciente ni su tratamiento. Respecto a la información brindada por los sujetos de estudio, se considera información de carácter personal y totalmente confidencial. Previo al uso y aplicación del instrumento, se realizó firma de consentimiento informado a los participantes, así como se llevó a cabo firma de documento de confidencialidad entre las partes que hacen parte del proyecto de investigación.

\section{Resultados}

El total de participantes que cumplió con los criterios de inclusión fue de 97 residentes de un total de 130 de los 5 programas de medicina familiar de Bogotá. Los resultados de la intervención se presentan de acuerdo con el mecanismo de evaluación del instrumento aplicado, que está subdividido en 5 categorías: a) datos sociodemográficos, b) hábitos y comportamientos, c) ambiente universitario, d) conocimientos mínimos sobre $\mathrm{AF}$ y e) práctica clínica.

\section{Datos sociodemográficos}

Dentro de la distribución de participantes de los diferentes programas, se encontró un 40,4 $\%$ correspondiente al primer año; un 31,3\%, a segundo año, y un $28,2 \%$, a tercer año de residencia. En cuanto a la autopercepción del estado de salud, el $9 \%$ lo considera excelente; el $81,8 \%$, bueno o muy bueno, y un $9 \%$, regular. El promedio de estatura fue de $166 \mathrm{~cm}$ y de peso fue de $66 \mathrm{~kg}$, con un promedio de índice de masa corporal (IMC) de 23. Se encontraron 5 participantes en sobrepeso (IMC > 25). Del total de participantes, el 76,7 \% se ubicó en los estratos socioeconómicos 3 y 4 (tabla 1).

Tabla 1.Datos sociodemográficos

\begin{tabular}{|c|c|}
\hline \multicolumn{2}{|c|}{ Programa de residencia } \\
\hline Código ID & Núm. participantes \\
\hline 1 & 13 \\
\hline 2 & 17 \\
\hline 3 & 33 \\
\hline 4 & 21 \\
\hline 5 & 13 \\
\hline Total & 97 \\
\hline \multicolumn{2}{|c|}{ Año de residencia } \\
\hline Año & Núm. participantes \\
\hline 1 & $40(40,4 \%)$ \\
\hline 2 & $31(31,31 \%)$ \\
\hline 3 & $28(28,28 \%)$ \\
\hline \multicolumn{2}{|c|}{ Nivel socioeconómico } \\
\hline Estrato & Núm. participantes \\
\hline 1 & 0 \\
\hline 2 & $6(6,06 \%)$ \\
\hline 3 & $27(27,27 \%)$ \\
\hline 4 & $49(49,49 \%)$ \\
\hline 5 & $12(12,12 \%)$ \\
\hline 6 & $5(5,05 \%)$ \\
\hline \multicolumn{2}{|c|}{ Autopercepción estado de salud } \\
\hline Excelente & $9(9,09 \%)$ \\
\hline Muy bueno & $40(40,40 \%)$ \\
\hline Bueno & $41(41,41 \%)$ \\
\hline Regular & $9(9,09 \%)$ \\
\hline Malo & 0 \\
\hline \multicolumn{2}{|c|}{ Medidas antropométricas } \\
\hline Variable & Promedio \\
\hline Peso (kg) & 66 \\
\hline Talla $(\mathrm{cm})$ & 166 \\
\hline $\begin{array}{l}\text { Índice de masa } \\
\text { corporal }\end{array}$ & 23 \\
\hline
\end{tabular}

\section{Hábitos y comportamientos}

El 40,4\% reportó realizar AF vigorosa durante un día a la semana; el $27 \%$, ningún día; el $14 \%$, dos días; el $9 \%$, tres días, y otro $9 \%$, más de 3 días. En cuanto al tiempo/semana dedicado a AF vigorosa, el $27 \%$ cumple con la recomendación mundial de mínimo $75 \mathrm{~min} /$ 
semana [11]. En cuanto a AF moderada, el 36,6 \% refirió realizarla un día a la semana; $22,2 \%$, ningún día; el $16 \%$, dos días; el $9 \%$, tres días, y el $16 \%$, más de tres días. El 12,1\% cumple con la recomendación internacional de mínimo 150 min/semana [11]. Ajustado a la recomendación internacional de realización combinada de AF vigorosa y moderada mínimo durante $150 \mathrm{~min} /$ semana, el 32,3\% cumple con esta [11]. El 47.4 $\%$ respondió realizar $\mathrm{AF}$ leve siete días a la semana; el $17 \%$, seis días; el $6 \%, 5$ y 4 días cada uno; el $8 \%$, tres días, y el $15 \%$, entre uno y dos días a la semana.

Las cuatro barreras principales percibidas para la realización de AF fueron: "falta de tiempo" (59 $\%)$, "falta de energía" (14\%), "falta de apoyo social" (8\%) y porque "no le gusta" (8\%). El tiempo promedio invertido viendo televisión o en videojuegos en el total de los participantes fue de 2,36 horas por semana (desviación estándar: 2,215).

\section{Ambiente universitario}

Frente a la pregunta "En general, imi universidad me ha motivado a llevar una vida sana?" el 44,3 \% estuvo fuertemente de acuerdo o de acuerdo; el $31,3 \%$, ni de acuerdo ni en desacuerdo, y el 24,2 $\%$, en desacuerdo o fuertemente en desacuerdo. Frente a "Mi universidad anima a los residentes para que realicen ejercicio", el 44,4\% se muestra en desacuerdo; el 32,3\%, de acuerdo, y el 23,2 $\%$, neutral. Acerca de "Nuestros docentes son buenos modelos de estilo de vida saludable", el $40,4 \%$ se mantiene en una posición neutral frente a un 29,2\% que está en desacuerdo.

\section{Conocimientos mínimos sobre actividad física}

En cuanto a "iCon qué frecuencia semanal se debe realizar AF?", el 51,5\% refiere recomendar 5 días por semana; el 32,3\%, 3 días; el 9\%, 4 días, y el $7 \%$, entre 6 y 7 días. Para "iCuál es la intensidad recomendada para realizar actividad física en un adulto?", el 76,7\% reportó indicar intensidad moderada; el 20,2 \%, vigorosa; el $2 \%$, leve, y un $1 \%$, "no sabe". Sobre "Cuánto tiempo al día se recomienda de AF para los adultos en general", el 80,8 \% indica que mínimo 30 minutos al día; el $8 \%$, 20 minutos/día; el $5 \%, 45$ minutos/ día; el $4 \%$, mínimo una hora/día, y el $2 \%, 15$ minutos/día.

\section{Práctica clínica}

Sobre "¿Con qué frecuencia usted evalúa/ pregunta sobre AF a sus pacientes?", el 43,4 $\%$ reportó siempre realizarlo; el $44,4 \%$, casi siempre; el 11,1\%, ocasionalmente, y el $1 \%$, nunca. En cuanto a "iCómo evalúa/pregunta sobre AF a sus pacientes?", el 52,5\% refirió hacerlo a través de preguntas generales sobre la cantidad de AF; el 41,4\%, mediante preguntas específicas sobre la duración e intensidad; el 5 $\%$, con preguntas que hacen parte de la historia clínica electrónica, y el $1 \%$, otra forma sin ser especificada cuál. En la prescripción de AF, el 93,9\% respondió realizarlo siempre o casi siempre; el $5 \%$, algunas veces, y el $1 \%$, nunca. La forma más frecuente de recomendarla es a través de la consejería verbal ( $85 \%)$, por escrito (11\%), referencia a programas de AF (2\%) e interconsulta a otro personal médico $(1 \%)$. Frente a la afirmación "los médicos tienen la responsabilidad de promover adecuados niveles de AF en sus pacientes", el 96,9\% se encontró fuertemente de acuerdo y de acuerdo; el $2 \%$, neutral, y el $1 \%$, fuertemente en desacuerdo. Acerca de "si yo realizo ejercicio y estoy en buena condición física tendré la capacidad de aconsejar a mis pacientes de una forma más coherente y efectiva", el 91,9\% respondió estar fuertemente de acuerdo o de acuerdo; el $6 \%$, neutral, y el $2 \%$, en desacuerdo. En cuanto a "soy efectivo en ayudar a mis pacientes a ser físicamente activos", el 52,5\% se consideró fuertemente de acuerdo y de acuerdo; el $42 \%$, neutral; el $4 \%$, en desacuerdo, y el $1 \%$, fuertemente en desacuerdo.

\section{Discusión}

Es relevante que el actual estudio sea el primero hecho en Colombia con el objetivo de evaluar hábitos sobre AF y explorar su asociación con las 
recomendaciones dadas a los pacientes, además de abordar el personal en formación de posgrado con un significativo número de residentes y de reunir a la gran mayoría de programas académicos de formación de una especialidad médicoquirúrgica (medicina familiar) de instituciones de reconocimiento nacional e internacional (solo faltó incluir el programa de especialización de la Universidad del Valle y la Universidad del Cauca, Colombia).

A pesar de que la autopercepción sobre el estado general de salud es buena y solo cinco de los participantes en el estudio fueron encontrados en sobrepeso, llama la atención la pobre realización de AF, tanto en calidad como en cantidad de tiempo, lo que resalta que la $\mathrm{AF}$ más frecuentemente realizada es la de intensidad leve.

Uno de los hallazgos importantes dentro de la AF ha sido el establecer las barreras para su realización en el grupo a estudio, pues se encontró la "falta de tiempo y energía" como factores determinantes, hecho que podría correlacionarse con la distribución del tiempo que un estudiante de posgrado debe tener para cumplir con sus actividades curriculares y extracurriculares, además de no contar con espacios disponibles dentro de sus currículos. Esto se asocia con un hallazgo discordante dentro del abordaje del ambiente universitario, ya que en general los residentes perciben que la universidad los motiva para vivir estilos de vida saludable o permanecen neutrales frente a dicha afirmación; pero no encuentran el apoyo o el ánimo necesario para la realización de AF.

Tal hecho cobra relevancia, dado que el mayor número de residentes participantes en el estudio son de primer año, lo que nos permitiría inferir que el ser nuevos en el programa y la carga académica pueden influir, así como el desconocimiento de los espacios para el desarrollo de AF. Otro hallazgo relevante y que se relaciona con lo anterior es la afectación del role model de los docentes como ejemplo de hábitos de vida saludable y AF. Aunque los resultados en su mayoría dejan una posición neutral, podemos discutir dos probables razones: para los residentes es indiferente el ejemplo del instructor o el estilo de vida no es lo suficientemente impactante en la vida de los estudiantes, al punto de no modificar su estructura de pensamiento ni sus actitudes.

De manera contradictoria, ello no se relaciona directamente con las recomendaciones dadas a los pacientes y con los conocimientos sobre AF. Los resultados en esta materia reflejan una gran mayoría que de "rutina" indaga a sus pacientes sobre la frecuencia de realización de $\mathrm{AF}$, con un adecuado interrogatorio sobre cantidad, intensidad y duración; además de la prescripción del ejercicio de manera verbal. Este hallazgo, pese a ser positivo para el estudio, deja ver la necesidad imperiosa de la inclusión de la AF dentro del formato de historia clínica de las instituciones, así como en los sistemas de formulación y prescripción clínica, respaldado por iniciativas internacionales como Excercise is Medicine ${ }^{\circledR}$ y estudios internacionales que contemplan la AF como un signo vital $[9,10]$ y como un tratamiento que debe ser indicado por escrito, pues ha mostrado mayor eficacia y adherencia cuando se indica de esa manera [12]. Finalmente, en cuanto a conocimiento mínimo sobre la prescripción de AF, el porcentaje mayoritario se ajusta a lo establecido en las últimas recomendaciones de salud pública (150 $\mathrm{min} /$ semana-intensidad de moderada a vigorosa) $[9,10]$.

En nuestro país, se hace imperioso el reconocimiento de la AF y el ejercicio como signo vital y como tratamiento para múltiples patologías prevalentes en nuestro medio. Debe existir un compromiso para su reglamentación dentro de la historia clínica. También se hace importante el fortalecimiento de programas de capacitación y entrenamiento en prescripción de actividad física o la inclusión dentro de los currículos de formación de los programas de posgrado para especialidades de atención primaria; además, se hace necesario la creación de iniciativas que no solo eduquen, sino motiven y permitan al residente llevar un estilo de vida saludable en medio del ámbito académico y universitario, desarrollando como primer pilar el role model docente-estudiante.

Dentro de las limitaciones del estudio, no fue posible garantizar la veracidad de las respuestas 
de los residentes a través de la confrontación con grupos de pacientes que hubieran sido atendidos por los participantes del estudio. Además, el mayor número de participantes fueron residentes de primer año, y al no contar con una muestra suficientemente grande de residentes de tercer año, puede generar sesgos, ya que el tiempo de formación y experiencia de un año a otro difieren.

\section{Conclusiones}

Los resultados evidenciados en el presente estudio y comparados con la literatura internacional disponible (Reino Unido, Estados Unidos, Nueva Zelanda y Canadá) muestran que hay convergencia en la necesidad de capacitar médicos de atención primaria con habilidades y conocimientos suficientes para la correcta y objetiva evaluación y prescripción de AF y estructurar un modelo de atención en el que se incluya la AF dentro de la historia clínica.

La prescripción verbal de la AF va contra la recomendación mundial sobre la prescripción correcta de AF y permite inferir la necesidad de incluirla dentro la historia clínica actual como un signo vital y como tratamiento médico. Hay divergencia con respecto a los hallazgos internacionales, pues, aunque la tendencia era una relación directa entre no realización de $\mathrm{AF}$ y no recomendación, nuestro estudio ha corroborado un antiguo predicamento: "Predicamos pero no aplicamos", pues aunque los participantes en el estudio tienen buenos conocimientos generales sobre AF y reconocen la importancia de su papel como médicos de atención primaria en su prescripción, no la realizan al mismo nivel que la conocen y recomiendan.

\section{Referencias}

1. Morishita Y, Miki A, Okada M, et al. Exercise counseling of primary care physicians in metabolic syndrome and cardiovascular diseases is associated with their specialty and exercise habits. Int J Gen Med. 2014;7:277-83.
2. Duperly J. John Duperly. Medicina Interna [blog]. Disponible en: http://www.johndup erly.com.

3. Morton S, Denlse G. Effects of physical activity counseling in primary care. JAMA. 2001;286:677-87.

4. Bull F, Milton K. A process evaluation of a "physical activity pathway" in the primary care setting. BMC Public Health. 2010;10:1-9.

5. Allenspach E, Handschin M, Kutlar M, et al. Patient and physician acceptance of campaign approach to promoting physical activity: the "move for health" project. Swiss Med WKLY. 2007;137:292-9.

6. Centro Regional para América Latina. Exercise is medicine. s. 1.: American College of Sports Medicine; 2014.

7. Frank E, Rothenberg R, Lewis C, et al. Correlates of physicians' prevention - related practices. Arch Fam Med. 2009;9:1-9.

8. Duperly J, Lobelo F, Segura C, et al. The association between Colombian medical students' healthy personal habits and a positive attitude toward preventive counseling: cross - sectional analyses. BMC Public Health. 2009;9(218):1-7.

9. Frank E, Breyan J, Elon L. Physician disclosure of healthy personal behaviors improves credibility and ability to motivate. Arch Fam Med. 2000;9:287-90.

10. Collazos V, Pierre V, Waak A, et al. recomendaciones mundiales sobre actividad física para la salud. Washington: OMS; 2010.

11. Frank E, Chair V, Chief D, et al. Medical Students' self reported typical counseling practices are similar to those assessed with standardized patients. Med Gen Med. 2005;7:1-5.

12. Elley R, Kerse N, Arroll B, et al. Effectiveness of counseling patients on physical activity in general practice: cluster randomized controlled trial. Br Med J. 2003;326:793. 